Interfaces and Free Boundaries 14 (2012), 185-203

DOI 10.4171/IFB/279

\title{
Two-phase flow problem coupled with mean curvature flow
}

\author{
CHUN LIU \\ Department of Mathematics, The Penn State University, University Park, PA 16802 USA \\ E-mail: liu@math.psu.edu \\ NORIFUMI SATO \\ Furano H.S., Furano, Hokkaido 076-0011, Japan \\ E-mail: differ@mb.infosnow.ne.jp \\ YOSHIHIRO TONEGAWA \\ Department of Mathematics, Hokkaido University, Sapporo 060-0810, Japan \\ E-mail: tonegawa@math.sci.hokudai.ac.jp
}

[Received 12 February 2010 and in revised form 31 March 2012]

\begin{abstract}
We prove the existence of generalized solution for incompressible and viscous non-Newtonian twophase fluid flow for spatial dimension $d=2$ and 3. Separating two shear thickening fluids with power law viscosity strictly above critical growth $p=(d+2) / 2$, the phase boundary moves along with the fluid flow plus its mean curvature while exerting surface tension force to the fluid. An approximation scheme combining the Galerkin method and the phase field method is adopted.
\end{abstract}

2010 Mathematics Subject Classification: Primary 35R37; Secondary 35B25, 76A05, 76T99.

Keywords: Two-phase fluid, surface energy, varifold, phase field method

\section{Introduction}

In this paper we prove existence results for a problem on incompressible viscous two-phase fluid flow in the torus $\Omega=\mathbb{T}^{d}=(\mathbb{R} / \mathbb{Z})^{d}, d=2$, 3. A freely moving $(d-1)$-dimensional phase boundary $\Gamma(t)$ separates the domain $\Omega$ into two domains $\Omega^{+}(t)$ and $\Omega^{-}(t), t \geqslant 0$. The fluid flow is described by means of the velocity field $u: \Omega \times[0, \infty) \rightarrow \mathbb{R}^{d}$ and the pressure $\Pi: \Omega \times[0, \infty) \rightarrow \mathbb{R}$. We assume the stress tensor of the fluids is of the form $T^{ \pm}(u, \Pi)=\tau^{ \pm}(e(u))-\Pi I$ on $\Omega^{ \pm}(t)$, respectively. Here $e(u)$ is the symmetric part of the velocity gradient $\nabla u$, i.e., $e(u)=(\nabla u+$ $\left.\nabla u^{T}\right) / 2$ and $I$ is the $d \times d$ identity matrix. Let $\mathbb{S}(d)$ be the set of $d \times d$ symmetric matrices. We assume that the functions $\tau^{ \pm}: \mathbb{S}(d) \rightarrow \mathbb{S}(d)$ is locally Lipschitz and satisfy for some $v_{0}>0$ and $p>\frac{d+2}{2}$ and for all $s, \hat{s} \in \mathbb{S}(d)$

$$
\begin{gathered}
v_{0}|s|^{p} \leqslant \tau^{ \pm}(s): s \leqslant v_{0}^{-1}\left(1+|s|^{p}\right), \\
\left|\tau^{ \pm}(s)\right| \leqslant v_{0}^{-1}\left(1+|s|^{p-1}\right), \\
\left(\tau^{ \pm}(s)-\tau^{ \pm}(\hat{s})\right):(s-\hat{s}) \geqslant 0 .
\end{gathered}
$$

Here we define $A: B=\operatorname{tr}(\mathrm{AB})$ for $d \times d$ matrices $A, B$. A typical example is $\tau^{ \pm}(s)=\left(a^{ \pm}+\right.$ $\left.b^{ \pm}|s|^{2}\right)^{\frac{p-2}{2}} s$ with $a^{ \pm}>0$ and $b^{ \pm}>0$. When the power $p$ of growth condition is larger than 2 , the fluid is called shear thickening fluid in general. 
We assume that the velocity field $u(x, t)$ satisfies the following non-Newtonian fluid flow equation:

$$
\begin{gathered}
\frac{\partial u}{\partial t}+u \cdot \nabla u=\operatorname{div}\left(T^{+}(u, \Pi)\right), \quad \operatorname{div} u=0 \quad \text { on } \Omega^{+}(t), t>0, \\
\frac{\partial u}{\partial t}+u \cdot \nabla u=\operatorname{div}\left(T^{-}(u, \Pi)\right), \quad \operatorname{div} u=0 \quad \text { on } \Omega^{-}(t), t>0, \\
u^{+}=u^{-}, \quad n \cdot\left(T^{+}(u, \Pi)-T^{-}(u, \Pi)\right)=\kappa_{1} H \quad \text { on } \Gamma(t), t>0 .
\end{gathered}
$$

The upper script \pm in (1.6) indicates the limiting values approaching to $\Gamma(t)$ from $\Omega^{ \pm}(t)$, respectively, $n$ is the unit outer normal vector of $\partial \Omega^{+}(t), H$ is the mean curvature vector of $\Gamma(t)$ and $\kappa_{1}>0$ is a constant. The condition (1.6) represents the force balance with an isotropic surface tension effect of the phase boundary. The boundary $\Gamma(t)$ is assumed to move with the velocity given by

$$
V_{\Gamma}=(u \cdot n) n+\kappa_{2} H \quad \text { on } \quad \Gamma(t), \quad t>0,
$$

where $\kappa_{2}>0$ is a constant. This differs from the conventional kinematic condition $\left(\kappa_{2}=0\right)$ and is motivated by the phase boundary motion with hydrodynamic interaction. The reader is referred to [31] and the references therein for the relevant physical background. By setting $\varphi=1$ on $\Omega^{+}(t)$, $\varphi=-1$ on $\Omega^{-}(t)$ and

$$
\tau(\varphi, e(u))=\frac{1+\varphi}{2} \tau^{+}(e(u))+\frac{1-\varphi}{2} \tau^{-}(e(u))
$$

on $\Omega^{+}(t) \cup \Omega^{-}(t)$, the equations (1.4)-(1.6) are expressed in the distributional sense as

$$
\begin{aligned}
\frac{\partial u}{\partial t}+u \cdot \nabla u & =\operatorname{div} \tau(\varphi, e(u))-\nabla \Pi+\kappa_{1} H \mathcal{H}^{d-1}\left\lfloor_{\Gamma(t)} \quad \text { on } \Omega \times(0, \infty),\right. \\
\operatorname{div} u & =0 \quad \text { on } \Omega \times(0, \infty) .
\end{aligned}
$$

where $\mathcal{H}^{d-1}$ is the $(d-1)$-dimensional Hausdorff measure. The expression (1.8) makes it evident that the phase boundary exerts surface tension force on the fluid wherever $H \neq 0$ on $\Gamma(t)$. Note that if $\Gamma(t)$ is a boundary of convex domain, the sign of $H$ is taken so that the presence of surface tension tends to accelerate the fluid flow inwards in general. We remark that the sufficiently smooth solutions of (1.4)-(1.7) satisfy the following energy equality,

$$
\frac{d}{d t}\left\{\frac{1}{2} \int_{\Omega}|u|^{2} d x+\kappa_{1} \mathcal{H}^{d-1}(\Gamma(t))\right\}=-\int_{\Omega} \tau(\varphi, e(u)): e(u) d x-\kappa_{1} \kappa_{2} \int_{\Gamma(t)}|H|^{2} d \mathcal{H}^{d-1} .
$$

This follows from the first variation formula for the surface measure

$$
\frac{d}{d t} \mathcal{H}^{d-1}(\Gamma(t))=-\int_{\Gamma(t)} V_{\Gamma} \cdot H d \mathcal{H}^{d-1}
$$

and by the equations (1.4)-(1.7).

The aim of the present paper is to prove the time-global existence of the weak solution for (1.4)-(1.7) (see Theorem 2.3 for the precise statement). We construct the approximate solution via the Galerkin method and the phase field method. Note that it is not even clear for our problem 
if the phase boundary may stay as a codimension 1 object since a priori irregular flow field may tear apart or crumble the phase boundary immediately, with a possibility of developing singularities and fine-scale complexities. Even if we set the initial datum to be sufficiently regular, the eventual occurrence of singularities of phase boundary or flow field may not be avoided in general. To accommodate the presence of singularities of phase boundary, we use the notion of varifolds from geometric measure theory. In establishing (1.7) we adopt the formulation due to Brakke [14] where he proved the existence of moving varifolds by mean curvature. We have the extra transport effect $(u \cdot n) n$ which is not very regular in the present problem. Typically we would only have $u \in L_{l o c}^{p}\left([0, \infty) ; W^{1, p}(\Omega)^{d}\right)$. This poses a serious difficulty in modifying Brakke's original construction in [14] which is already intricate and involved. Instead we take advantage of the recent progress on the understanding on the Allen-Cahn equation with transport term to approximate the motion law (1.7),

$$
\frac{\partial \varphi}{\partial t}+u \cdot \nabla \varphi=\kappa_{2}\left(\Delta \varphi-\frac{W^{\prime}(\varphi)}{\varepsilon^{2}}\right)
$$

Here $W$ is an equal depth double-well potential and we set $W(\varphi)=\left(1-\varphi^{2}\right)^{2} / 2$. When $\varepsilon \rightarrow 0$, we have proved in [30] that the interface moves according to the velocity (1.7) in the sense of Brakke with a suitable regularity assumptions on $u$. To be more precise, we use a regularized version of (ACT) as we present later for the result of [30] to be applicable. The result of [30] was built upon those of many earlier works, most relevant being [24, 25] which analyzed (ACT) with $u=0$, and also [23, 38, 39, 44]. On some technical side, we briefly mention why we need the restriction $p>\frac{d+2}{2}$. Note that (1.1) via Korn's inequality gives a control of $L^{p}$ norm of $\nabla u$. In terms of scaling, $p=\frac{d+2}{2}$ corresponds to the scale invariant power for $\|\nabla u\|_{L^{p}\left([0, \infty) ; L^{p}\left(\mathbb{R}^{d}\right)\right)}$ under the natural change of variables $\tilde{x}=x / \lambda, \tilde{t}=t / \lambda^{2}, \tilde{u}=\lambda u$ for $\lambda>0$. In analyzing the local behavior of phase boundary, it is essential that the transport term $u$ may be regarded as a perturbative term for surface measure of phase boundary. Having $p>\frac{d+2}{2}$ makes the mean curvature flow more dominant than the transport effect due to $u$.

Since the literature of two-phase flow is immense and continues to grow rapidly, we mention results which are closely related or whose aims point to some time-global existence with general initial data. In the case without surface tension $\left(\kappa_{1}=\kappa_{2}=0\right)$, Solonnikov [41] proved the time-local existence of classical solution. The time-local existence of weak solution was proved by Solonnikov [42], Beale [8], Abels [1], and others. For the time-global existence of weak solution, Beale [9] proved in the case that the initial data is small. Nouri-Poupaud [36] considered the case of multi-phase fluid. Giga-Takahashi [20] considered the problem within the framework of level set method. When $\kappa_{1}>0, \kappa_{2}=0$, Plotnikov [37] proved the time-global existence of varifold solution for $d=2, p>2$, and Abels [2] proved the time-global existence of measure-valued solution for $d=2,3, p>\frac{2 d}{d+2}$. When $\kappa_{1}>0, \kappa_{2}>0$, Maekawa [32] proved the time-local existence of classical solution with $p=2$ (Navier-Stokes and Stokes) and for all dimension. As for related phase field approximations of sharp interface model which we adopt in this paper, Liu and Walkington [31] considered the case of fluids containing visco-hyperelastic particles. Perhaps the most closely related work to the present paper is that of Mugnai and Röger [35] which studied the identical problem with $p=2$ (linear viscosity case) and $d=2,3$. There they introduced the notion of $L^{2}$ velocity and showed that (1.7) is satisfied in a weak sense different from that of Brakke for the limiting interface. Soner [43] dealt with a coupling of Allen-Cahn and heat equations to approximate the Mullins-Sekerka problem with kinetic undercooling. Soner's work is closely related in that he showed the surface energy density bound which is also essential in the present problem. While the 
present article discussed the coupling of the Allen-Cahn equation and non-Newtonian flow equation, there has been a vast literature dealing with a coupling of the Cahn-Hilliard equation and the NavierStokes equation (the so called Model $\mathrm{H}$ [21]) in recent years. Note that the latter coupling has the advantage that the order parameter $\varphi$ becomes a conserved quantity. On the other hand, due to the fact that the Cahn-Hilliard equation is a 4th order equation, the analogous measure-theoretic analysis of sharp phase boundary for weak solutions poses serious difficulties. For this coupling problem we mention [3-5, 7, 10-13, 18, 19, 26, 45] and references therein among numerous papers.

The organization of this paper is as follows. In Section 2, we summarize the basic notations and main results. In Section 3 we construct a sequence of approximating solutions for the two-phase flow problem. In Section 4 we first recall the result of [30] which establishes the upper density ratio bound for surface energy and which proves (1.7). We then combine the results from Section 3 to obtain the desired weak solution for the two-phase flow problem.

\section{Preliminaries and main results}

For $d \times d$ matrices $A, B$ we denote $A: B=\operatorname{tr}(A B)$ and $|A|:=\sqrt{A: A}$. For $a \in \mathbb{R}^{d}$, we denote by $a \otimes a$ the $d \times d$ matrix with the $i$-th row and $j$-th column entry equal to $a_{i} a_{j}$.

\section{$2.1 \quad$ Function spaces}

Set $\Omega=\mathbb{T}^{d}$ throughout this paper. We set function spaces for $p>\frac{d+2}{2}$ and $s \in \mathbb{Z}^{+} \cup\{0\}$ as follows:

$$
\begin{gathered}
V=\left\{v \in C^{\infty}(\Omega)^{d} ; \operatorname{div} v=0\right\}, \\
W^{s, p}(\Omega)=\left\{v: \nabla^{j} v \in L^{p}(\Omega) \text { for } 0 \leqslant j \leqslant s\right\}, \\
V^{s, p}=\text { closure of } v \text { in the } W^{s, p}(\Omega)^{d} \text {-norm. }
\end{gathered}
$$

We denote the dual space of $V^{s, p}$ by $\left(V^{s, p}\right)^{*}$. The $L^{2}$ inner product is denoted by $(\cdot, \cdot)$. Let $\chi_{A}$ be the characteristic function of $A$, and for $\chi_{A} \in B V(\Omega)$ let $\left|\nabla \chi_{A}\right|$ be the total variation measure of the distributional derivative $\nabla \chi_{A}$.

\subsection{Varifold notations}

We recall some notions from geometric measure theory and refer to [6, 14, 40] for more details. A general $k$-varifold in $\mathbb{R}^{d}$ is a Radon measure on $\mathbb{R}^{d} \times G(d, k)$, where $G(d, k)$ is the space of $k$-dimensional subspaces in $\mathbb{R}^{d}$. We denote the set of all general $k$-varifolds by $\mathbf{V}_{k}\left(\mathbb{R}^{d}\right)$. When $S$ is a $k$-dimensional subspace, we also use $S$ to denote the orthogonal projection matrix corresponding to $\mathbb{R}^{d} \rightarrow S$. The first variation of $V$ can be written as

$$
\delta V(g)=\int_{\mathbb{R}^{d} \times G(d, k)} \nabla g(x): S d V(x, S)=-\int_{\mathbb{R}^{d}} g(x) \cdot H(x) d\|V\|(x) \quad \text { if }\|\delta V\| \ll\|V\| .
$$

Here $V \in \mathbf{V}_{k}\left(\mathbb{R}^{d}\right),\|V\|$ is the mass measure of $V, g \in C_{c}^{1}\left(\mathbb{R}^{d}\right)^{d}, H=H_{V}$ is the generalized mean curvature vector if it exists and $\|\delta V\| \ll\|V\|$ denotes that $\|\delta V\|$ is absolutely continuous with respect to $\|V\|$. 
We call a Radon measure $\mu k$-integral if $\mu$ is represented as $\mu=\left.\theta \mathcal{H}^{k}\right|_{X}$, where $X$ is a countably $k$-rectifiable, $\mathcal{H}^{k}$-measurable set, and $\theta \in L_{\text {loc }}^{1}\left(\mathcal{H}^{k}\left\lfloor_{X}\right)\right.$ is positive and integer-valued $\mathcal{H}^{k}$ a.e on $X$. $\mathcal{H}^{k}\left\lfloor x\right.$ denotes the restriction of $\mathcal{H}^{k}$ to the set $X$. We denote the set of $k$-integral Radon measures by $\ell m_{k}$. We say that a $k$-integral varifold is of unit density if $\theta=1 \mathcal{H}^{k}$ a.e. on $X$. For each such $k$-integral measure $\mu$ corresponds a unique $k$-varifold $V$ defined by

$$
\int_{\mathbb{R}^{d} \times G(d, k)} \phi(x, S) d V(x, S)=\int_{\mathbb{R}^{d}} \phi\left(x, T_{x} \mu\right) d \mu(x) \quad \text { for } \phi \in C_{c}\left(\mathbb{R}^{d} \times G(d, k)\right),
$$

where $T_{x} \mu$ is the approximate tangent $k$-plane. Note that $\mu=\|V\|$. We make such identification in the following. For this reason we define $H_{\mu}$ as $H_{V}$ (or simply $H$ ) if the latter exists. When $X$ is a $C^{2}$ submanifold without boundary and $\theta$ is constant on $X, H$ corresponds to the usual mean curvature vector for $X$. In the following we suitably adopt the above notions on $\Omega=\mathbb{T}^{d}$ such as $\mathbf{V}_{k}(\Omega)$, which present no essential difficulties.

\subsection{Weak formulation of free boundary motion}

For sufficiently smooth surface $\Gamma(t)$ moving by the velocity (1.7), the following holds for any $\phi \in C^{2}\left(\Omega ; \mathbb{R}^{+}\right)$due to the first variation formula (1.10):

$$
\frac{d}{d t} \int_{\Gamma(t)} \phi d \mathcal{H}^{d-1} \leqslant \int_{\Gamma(t)}(-\phi H+\nabla \phi) \cdot\left\{\kappa_{2} H+(u \cdot n) n\right\} d \mathcal{H}^{d-1} .
$$

One can check that having this inequality for any $\phi \in C^{2}\left(\Omega ; \mathbb{R}^{+}\right)$implies (1.7) thus (2.1) is equivalent to (1.7). Such use of non-negative test functions to characterize the motion law is due to Brakke [14] where he developed the theory of varifolds moving by the mean curvature. Here we suitably modify Brakke's approach to incorporate the transport term $u$. To do this we recall

THEOREM 2.1 (Meyers-Ziemer inequality) For any Radon measure $\mu$ on $\mathbb{R}^{d}$ with

$$
D=\sup _{r>0, x \in \mathbb{R}^{d}} \frac{\mu\left(B_{r}(x)\right)}{\omega_{d-1} r^{d-1}}<\infty
$$

we have

$$
\int_{\mathbb{R}^{d}}|\phi| d \mu \leqslant c D \int_{\mathbb{R}^{d}}|\nabla \phi| d x
$$

for $\phi \in C_{c}^{1}\left(\mathbb{R}^{d}\right)$. Here $c$ depends only on $d$.

See [34] and [46, p. 266]. By localizing (2.2) to $\Omega=\mathbb{T}^{d}$ we obtain (with $r$ in the definition of $D$ above replaced by $0<r<1 / 2$ )

$$
\int_{\Omega}|\phi|^{2} d \mu \leqslant c D\left(\|\phi\|_{L^{2}(\Omega)}^{2}+\|\nabla \phi\|_{L^{2}(\Omega)}^{2}\right),
$$

where the constant $c$ may be different due to the localization but depends only on $d$. The inequality (2.3) allows us to define $\int_{\Omega}|\phi|^{2} d \mu$ for $\phi \in W^{1,2}(\Omega)$ by the standard density argument when $D<\infty$. 
We define for any Radon measure $\mu, u \in L^{2}(\Omega)^{d}$ and $\phi \in C^{1}\left(\Omega: \mathbb{R}^{+}\right)$

$$
\mathrm{B}(\mu, u, \phi)=\int_{\Omega}(-\phi H+\nabla \phi) \cdot\left\{\kappa_{2} H+(u \cdot n) n\right\} d \mu
$$

if $\mu \in \mathbb{l}_{d-1}(\Omega)$ with generalized mean curvature $H \in L^{2}(\mu)^{d}$ and with

$$
\sup _{\frac{1}{2}>r>0, x \in \Omega} \frac{\mu\left(B_{r}(x)\right)}{\omega_{d-1} r^{d-1}}<\infty
$$

and $u \in W^{1,2}(\Omega)^{d}$. Due to the definition of $l m_{d-1}(\Omega)$, the unit normal vector $n$ is uniquely defined $\mu$ a.e. on $\Omega$ modulo \pm sign. Since we have $(u, n) n$ in (2.4), the choice of sign does not affect the definition. The right-hand side of (2.4) gives a well-defined finite value due to the stated conditions and (2.3). If any one of the conditions is not satisfied, we define $B(\mu, u, \phi)=-\infty$.

Next we note

Proposition 2.2 For any $0<T<\infty$ and $p>\frac{d+2}{2}$,

$$
\left\{u \in L^{p}\left([0, T] ; V^{1, p}\right) ; \frac{\partial u}{\partial t} \in L^{\frac{p}{p-1}}\left([0, T] ;\left(V^{1, p}\right)^{*}\right)\right\} \hookrightarrow C\left([0, T] ; V^{0,2}\right) .
$$

The Sobolev embedding gives $V^{1, p} \hookrightarrow V^{0,2}$ for such $p$ and we may apply [33, p. 35, Lemma 2.45] to obtain the above embedding. Indeed, we only need $p>\frac{2 d}{d+2}$ for Proposition 2.2 to hold and we have $\frac{d+2}{2}>\frac{2 d}{d+2}$. Thus for this class of $u$ we may define $u(\cdot, t) \in V^{0,2}$ for all $t \in[0, T]$ instead of a.e. $t$ and we may tacitly assume that we redefine $u$ in this way for all $t$. For $\left\{\mu_{t}\right\}_{t \in[0, \infty)}$, $u \in L_{l o c}^{p}\left([0, \infty) ; V^{1, p}\right)$ with $\frac{\partial u}{\partial t} \in L_{l o c}^{\frac{p}{p-1}}\left([0, \infty) ;\left(V^{1, p}\right)^{*}\right)$ for $p>\frac{d+2}{2}$ and $\phi \in C^{1}\left(\Omega ; \mathbb{R}^{+}\right)$, we define $B\left(\mu_{t}, u(\cdot, t), \phi\right)$ as in $(2.4)$ for all $t \geqslant 0$.

\subsection{The main results}

Our main results are the following.

THEOREM 2.3 Let $d=2$ or 3 and $p>\frac{d+2}{2}$. Let $\Omega=\mathbb{T}^{d}$. Assume that locally Lipschitz functions $\tau^{ \pm}: \mathbb{S}(d) \rightarrow \mathbb{S}(d)$ satisfy (1.1)-(1.3). For any initial data $u_{0} \in V^{0,2}$ and $\Omega^{+}(0) \subset \Omega$ having $C^{1}$ boundary $\partial \Omega^{+}(0)$, there exist

(a) $u \in L^{\infty}\left([0, \infty) ; V^{0,2}\right) \cap L_{l o c}^{p}\left([0, \infty) ; V^{1, p}\right)$ with $\frac{\partial u}{\partial t} \in L_{l o c}^{\frac{p}{p-1}}\left([0, \infty) ;\left(V^{1, p}\right)^{*}\right)$,

(b) a family of Radon measures $\left\{\mu_{t}\right\}_{t \in[0, \infty)}$ with $\mu_{t} \in \mathcal{l} m_{d-1}$ for a.e. $t \in[0, \infty)$ and

(c) $\varphi \in B V_{l o c}(\Omega \times[0, \infty)) \cap C_{l o c}^{\frac{1}{2}}\left([0, \infty) ; L^{1}(\Omega)\right)$ with $\sup _{t \in[0, \infty)}\|\varphi(\cdot, t)\|_{B V(\Omega)}<\infty$ such that the following properties hold:

(i) The triplet $\left(u(\cdot, t), \varphi(\cdot, t), \mu_{t}\right)_{t \in[0, \infty)}$ is a weak solution of (1.8). More precisely, for any $T>0$ we have

$$
\begin{aligned}
\int_{0}^{T} \int_{\Omega}-u \cdot \frac{\partial v}{\partial t}+(u \cdot \nabla u) \cdot v+\tau(\varphi, e(u)): e(v) d x d t & \\
& =\int_{\Omega} u_{0} \cdot v(0) d x+\int_{0}^{T} \int_{\Omega} \kappa_{1} H \cdot v d \mu_{t} d t
\end{aligned}
$$


for any $v \in C^{\infty}([0, T]$; $v)$ such that $v(T)=0$. Here $H \in L^{2}\left([0, \infty) ; L^{2}\left(\mu_{t}\right)^{d}\right)$ exists and is the generalized mean curvature vector corresponding to $\mu_{t}$.

(ii) The triplet $\left(u(\cdot, t), \varphi(\cdot, t), \mu_{t}\right)_{t \in[0, \infty)}$ satisfies the energy inequality

$$
\begin{array}{r}
\frac{1}{2} \int_{\Omega}|u(\cdot, T)|^{2} d x \\
+\kappa_{1} \mu_{T}(\Omega)+\int_{0}^{T} \int_{\Omega} \tau(\varphi, e(u)): e(u) d x d t+\kappa_{1} \kappa_{2} \int_{0}^{T} \int_{\Omega}|H|^{2} d \mu_{t} d t \\
\quad \leqslant \frac{1}{2} \int_{\Omega}\left|u_{0}\right|^{2} d x+\kappa_{1} \mathcal{H}^{d-1}\left(\partial \Omega^{+}(0)\right)=: E_{0}
\end{array}
$$

for all $T<\infty$.

(iii) For all $0 \leqslant t_{1}<t_{2}<\infty$ and $\phi \in C^{2}\left(\Omega ; \mathbb{R}^{+}\right)$we have

$$
\mu_{t_{2}}(\phi)-\mu_{t_{1}}(\phi) \leqslant \int_{t_{1}}^{t_{2}} \mathbb{B}\left(\mu_{t}, u(\cdot, t), \phi\right) d t .
$$

Moreover, $\mathbb{B}\left(\mu_{t}, u(\cdot, t), \phi\right) \in L_{l o c}^{1}([0, \infty))$.

(iv) We set $D_{0}=\sup _{0<r<1 / 2, x \in \Omega} \frac{\varkappa^{d-1}\left(\partial \Omega^{+}(0) \cap B_{r}(x)\right)}{\omega_{d-1} r^{d-1}}$. For any $0<T<\infty$, there exists a constant $D=D\left(E_{0}, D_{0}, T, p, \nu_{0}, \kappa_{1}, \kappa_{2}\right)$ such that

$$
\sup _{0<r<1 / 2, x \in \Omega} \frac{\mu_{t}\left(B_{r}(x)\right)}{\omega_{d-1} r^{d-1}} \leqslant D
$$

for all $t \in[0, T]$.

(v) The function $\varphi$ satisfies the following properties.

(1) $\varphi= \pm 1$ a.e. on $\Omega$ for all $t \in[0, \infty)$.

(2) $\varphi(x, 0)=\chi_{\Omega^{+}(0)}-\chi_{\Omega \backslash \Omega \Omega^{+}(0)}$ a.e. on $\Omega$.

(3) $\operatorname{spt}\left|\nabla \chi_{\{\varphi(\cdot, t)=1\}}\right| \subset \operatorname{spt} \mu_{t}$ for all $t \in[0, \infty)$.

(vi) There exists

$$
T_{1}=T_{1}\left(E_{0}, D_{0}, p, v_{0}, \kappa_{1}, \kappa_{2}\right)>0
$$

such that $\mu_{t}$ is of unit density for a.e. $t \in\left[0, T_{1}\right]$. In addition $\left|\nabla \chi_{\{\varphi(\cdot, t)=1\}}\right|=\mu_{t}$ for a.e. $t \in\left[0, T_{1}\right]$.

REMARK 2.4 Somewhat different from the case $u=0$ we do not expect that

$$
\limsup _{\Delta t \rightarrow 0} \frac{\mu_{t+\Delta t}(\phi)-\mu_{t}(\phi)}{\Delta t} \leqslant \mathbb{B}\left(\mu_{t}, u(\cdot, t), \phi\right)
$$

holds for all $t \geqslant 0$ and $\phi \in C^{2}\left(\Omega ; \mathbb{R}^{+}\right)$in general. While we know that the right-hand side is $<\infty$ (by definition) for all $t$, we do not know in general if the left-hand side is $<\infty$. One may even 
expect that at a time when $\int_{\Omega}|\nabla u(\cdot, t)|^{p} d x=\infty$, it may be $\infty$. Thus we may need to define (1.7) in the integral form (2.8) for the definition of Brakke's flow. Note that in case $u=0$, one can show that the left-hand side of (2.9) is $<\infty$ for all $t \geqslant 0$ (see [14]).

REMARK 2.5 The difficulty of multiplicities have been often encountered in the measure-theoretic setting like ours. Varifold solutions constructed by Brakke [14] have the same properties in this regard. On the other hand, (vi) says that there is no 'folding' for some initial time interval $\left[0, T_{1}\right]$ at least.

REMARK 2.6 In the following we set $\kappa_{1}=\kappa_{2}=1$ for notational simplicity, while all the argument can be modified with any positive $\kappa_{1}$ and $\kappa_{2}$ with no essential differences. On the other hand, their being positive plays an essential role, and most of the estimates and claims deteriorate as $\kappa_{1}, \kappa_{2} \rightarrow 0$ and fail in the limit. How severely they fail in the limit may be of independent interest which we do not pursue in the present paper. Note that $\kappa_{2}=0$ limit should correspond precisely to the setting of Plotnikov [37] for $d=2$.

We use the following theorem. See [33, p. 196] and the reference therein.

THEOREM 2.7 (Korn's inequality) Let $1<p<\infty$. Then there exists a constant $c_{K}=c(p, d)$ such that

$$
\|v\|_{W^{1, p}(\Omega)}^{p} \leqslant c_{K}\left(\|e(v)\|_{L^{p}(\Omega)}^{p}+\|v\|_{L^{1}(\Omega)}^{p}\right)
$$

holds for all $v \in W^{1, p}(\Omega)^{d}$.

\section{Existence of approximate solution}

In this section we construct a sequence of approximate solutions of (1.4)-(1.7) by the Galerkin method and the phase field method. The proof is a suitable modification of [28] for the nonNewtonian setting even though we need to incorporate a suitable smoothing of the interaction terms.

First we prepare a few definitions. We fix a sequence $\left\{\varepsilon_{i}\right\}_{i=1}^{\infty}$ with $\lim _{i \rightarrow \infty} \varepsilon_{i}=0$ and fix a radially symmetric non-negative function $\zeta \in C_{c}^{\infty}\left(\mathbb{R}^{d}\right)$ with $\operatorname{spt} \zeta \subset B_{1}(0)$ and $\int \zeta d x=1$. For a fixed $0<\gamma<\frac{1}{2}$ we define

$$
\zeta^{\varepsilon_{i}}(x)=\frac{1}{\varepsilon_{i}^{\gamma}} \zeta\left(\frac{x}{\varepsilon_{i}^{\gamma / d}}\right) .
$$

We defined $\zeta^{\varepsilon_{i}}$ so that $\int \zeta^{\varepsilon_{i}} d x=1,\left|\zeta^{\varepsilon_{i}}\right| \leqslant c(d) \varepsilon_{i}^{-\gamma}$ and $\left|\nabla \zeta^{\varepsilon_{i}}\right| \leqslant c(d) \varepsilon_{i}^{-\gamma-\gamma / d}$.

For a given initial data $\Omega^{+}(0) \subset \Omega$ with $C^{1}$ boundary $\partial \Omega^{+}(0)$, we can approximate $\Omega^{+}(0)$ in $C^{1}$ topology by a sequence of domains $\Omega^{i+}(0)$ with $C^{3}$ boundaries. Let $d^{i}(x)$ be the signed distance function to $\partial \Omega^{i+}(0)$ so that $d^{i}(x)>0$ on $\Omega^{i+}(0)$ and $d^{i}(x)<0$ on $\Omega^{i-}(0)$. Choose $b^{i}>$ 0 so that $d^{i}$ is $C^{3}$ function on the $b^{i}$-neighborhood of $\partial \Omega^{i+}(0)$. Now we associate $\left\{\varepsilon_{i}\right\}_{i=1}^{\infty}$ with $\Omega^{i+}(0)$ by re-labeling the index if necessary so that $\lim _{i \rightarrow \infty} \varepsilon_{i} / b^{i}=0$ and $\lim _{i \rightarrow \infty} \varepsilon_{i}^{j-1}\left|\nabla^{j} d^{i}\right|=$ 0 for $j=2,3$ on the $b^{i}$-neighborhood of $\partial \Omega^{i+}(0)$. Let $h \in C^{\infty}(\mathbb{R})$ be a function such that $h$ is monotone increasing, $h(s)=s$ for $0 \leqslant s \leqslant 1 / 4$ and $h(s)=1 / 2$ for $1 / 2<s$, and define $h(-s)=-h(s)$ for $s<0$. Then define

$$
\varphi_{0}^{\varepsilon_{i}}(x)=\tanh \left(b^{i} h\left(d^{i}(x) / b^{i}\right) / \varepsilon_{i}\right) .
$$


Note that we have $\varphi_{0}^{\varepsilon_{i}} \in C^{3}(\Omega)$ and $\varepsilon_{i}^{j}\left|\nabla^{j} \varphi_{0}^{\varepsilon_{i}}\right|$ for $j=1,2,3$ are bounded uniformly independent of $i$. The well-known property of phase field approximation shows that

$$
\lim _{i \rightarrow \infty}\left\|\varphi_{0}^{\varepsilon_{i}}-\left(\chi_{\Omega^{+}(0)}-\chi_{\Omega^{-}(0)}\right)\right\|_{L^{1}(\Omega)}=0, \quad \frac{1}{\sigma}\left(\frac{\varepsilon_{i}\left|\nabla \varphi_{0}^{\varepsilon_{i}}\right|^{2}}{2}+\frac{W\left(\varphi_{0}^{\varepsilon_{i}}\right)}{\varepsilon_{i}}\right) d x \rightarrow \mathcal{H}^{d-1}\left\lfloor_{\partial \Omega^{+}(0)}\right.
$$

as Radon measures. Here $\sigma=\int_{-1}^{+1} \sqrt{2 W(s)} d s$.

For $V^{s, 2}$ with $s>\frac{d}{2}+1$ let $\left\{\omega^{i}\right\}_{i=1}^{\infty}$ be a set of basis for $V^{s, 2}$ such that it is orthonormal in $V^{0,2}$. The choice of $s$ is made so that the Sobolev embedding theorem implies $W^{s-1,2}(\Omega) \hookrightarrow L^{\infty}(\Omega)$ thus $\nabla \omega^{i} \in L^{\infty}(\Omega)^{d^{2}}$.

Let $P_{i}: V^{0,2} \rightarrow V_{i}^{0,2}=\operatorname{span}\left\{\omega_{1}, \omega_{2}, \cdots, \omega_{i}\right\}$ be the orthogonal projection. We then project the problem (1.4)-(1.7) to $V_{i}^{0,2}$ by utilizing the orthogonality in $V^{0,2}$. Note that just as in [28], we approximate the mean curvature term in (1.8) by the appropriate phase field approximation. We consider the following problem:

$$
\begin{aligned}
\frac{\partial u^{\varepsilon_{i}}}{\partial t}=P_{i}\left(\operatorname{div} \tau\left(\varphi^{\varepsilon_{i}}, e\left(u^{\varepsilon_{i}}\right)\right)-u^{\varepsilon_{i}} \cdot \nabla u^{\varepsilon_{i}}\right. & \\
\left.-\frac{\varepsilon_{i}}{\sigma} \operatorname{div}\left(\left(\nabla \varphi^{\varepsilon_{i}} \otimes \nabla \varphi^{\varepsilon_{i}}\right) * \zeta^{\varepsilon_{i}}\right)\right) & \text { on } \Omega \times[0, \infty), \\
u^{\varepsilon_{i}}(\cdot, t) \in V_{i}^{0,2} & \text { for } t \geqslant 0, \\
\frac{\partial \varphi^{\varepsilon_{i}}}{\partial t}+\left(u^{\varepsilon_{i}} * \zeta^{\varepsilon_{i}}\right) \cdot \nabla \varphi^{\varepsilon_{i}}=\Delta \varphi^{\varepsilon_{i}}-\frac{W^{\prime}\left(\varphi^{\varepsilon_{i}}\right)}{\varepsilon_{i}^{2}} & \text { on } \Omega \times[0, \infty), \\
u^{\varepsilon_{i}}(x, 0)=P_{i} u_{0}(x), \quad \varphi^{\varepsilon_{i}}(x, 0)=\varphi_{0}^{\varepsilon_{i}}(x) & \text { on } \Omega .
\end{aligned}
$$

Here $*$ is the usual convolution. We prove the following theorem.

THEOREM 3.1 For any $i \in \mathbb{N}, u_{0} \in V^{0,2}$ and $\varphi_{0}^{\varepsilon_{i}}$, there exists a weak solution $\left(u^{\varepsilon_{i}}, \varphi^{\varepsilon_{i}}\right)$ of (3.4)(3.7) such that $u^{\varepsilon_{i}} \in L^{\infty}\left([0, \infty) ; V^{0,2}\right) \cap L_{l o c}^{p}\left([0, \infty) ; V^{1, p}\right),\left|\varphi^{\varepsilon_{i}}\right| \leqslant 1, \varphi^{\varepsilon_{i}} \in L^{\infty}\left([0, \infty) ; C^{3}(\Omega)\right)$ and $\frac{\partial \varphi^{\varepsilon_{i}}}{\partial t} \in L^{\infty}\left([0, \infty) ; C^{1}(\Omega)\right)$.

We write the above system in terms of $u^{\varepsilon_{i}}=\sum_{k=1}^{i} c_{k}^{\varepsilon_{i}}(t) \omega_{k}(x)$ first. Since

$$
\begin{gathered}
\left(\frac{d}{d t} u^{\varepsilon_{i}}, \omega_{j}\right)=\left(\frac{d}{d t} \sum_{k=1}^{i} c_{k}^{\varepsilon_{i}}(t) \omega_{k}, \omega_{j}\right)=\frac{d}{d t} c_{j}^{\varepsilon_{i}}(t), \\
\left(u^{\varepsilon_{i}} \cdot \nabla u^{\varepsilon_{i}}, \omega_{j}\right)=\sum_{k, l=1}^{i} c_{k}^{\varepsilon_{i}}(t) c_{l}^{\varepsilon_{i}}(t)\left(\omega_{k} \cdot \nabla \omega_{l}, \omega_{j}\right), \\
\varepsilon_{i}\left(\operatorname{div}\left(\left(\nabla \varphi^{\varepsilon_{i}} \otimes \nabla \varphi^{\varepsilon_{i}}\right) * \zeta^{\varepsilon_{i}}\right), \omega_{j}\right)=-\varepsilon_{i} \int_{\Omega}\left(\nabla \varphi^{\varepsilon_{i}} \otimes \nabla \varphi^{\varepsilon_{i}}\right) * \zeta^{\varepsilon_{i}}: \nabla \omega_{j} d x, \\
\left(\operatorname{div} \tau\left(\varphi^{\varepsilon_{i}}, e\left(u^{\varepsilon_{i}}\right)\right), \omega_{j}\right)=-\int_{\Omega} \tau\left(\varphi^{\varepsilon_{i}}, e\left(u^{\varepsilon_{i}}\right)\right): e\left(\omega_{j}\right) d x
\end{gathered}
$$


for $j=1, \cdots, i,(3.4)$ is equivalent to

$$
\begin{aligned}
\frac{d}{d t} c_{j}^{\varepsilon_{i}}(t) & =-\int_{\Omega} \tau\left(\varphi^{\varepsilon_{i}}, e\left(u^{\varepsilon_{i}}\right)\right): e\left(\omega_{j}\right) d x-\sum_{k, l=1}^{i} c_{k}^{\varepsilon_{i}}(t) c_{l}^{\varepsilon_{i}}(t)\left(\omega_{k} \cdot \nabla \omega_{l}, \omega_{j}\right) \\
+ & \frac{\varepsilon_{i}}{\sigma} \int_{\Omega}\left(\nabla \varphi^{\varepsilon_{i}} \otimes \nabla \varphi^{\varepsilon_{i}}\right) * \zeta^{\varepsilon_{i}}: \nabla \omega_{j} d x=A_{j}^{\varepsilon_{i}}(t)+B_{k l j} c_{k}^{\varepsilon_{i}}(t) c_{l}^{\varepsilon_{i}}(t)+D_{j}^{\varepsilon_{i}}(t) .
\end{aligned}
$$

Moreover, the initial condition of $c_{j}^{\varepsilon_{i}}$ is

$$
c_{j}^{\varepsilon_{i}}(0)=\left(u_{0}, \omega_{j}\right) \text { for } j=1,2, \ldots, i .
$$

We also set

$$
E_{0}=\mathcal{H}^{d-1}\left(\partial \Omega^{+}(0)\right)+\frac{1}{2} \int_{\Omega}\left|u_{0}\right|^{2} d x
$$

and note that

$$
\frac{1}{\sigma} \int_{\Omega}\left(\frac{\varepsilon_{i}\left|\nabla \varphi_{0}^{\varepsilon_{i}}\right|^{2}}{2}+\frac{W\left(\varphi_{0}^{\varepsilon_{i}}\right)}{\varepsilon_{i}}\right) d x+\frac{1}{2} \sum_{j=1}^{i}\left(c_{j}^{\varepsilon_{i}}(0)\right)^{2} \leqslant E_{0}+o(1)
$$

by (3.3) and by the projection $P_{i}$ being orthonormal.

We use the following lemma to prove Theorem 3.1.

LEMMA 3.2 There exists a constant $T_{0}=T_{0}\left(E_{0}, i, v_{0}, p\right)>0$ such that (3.4)-(3.7) with (3.9) has a weak solution $\left(u^{\varepsilon_{i}}, \varphi^{\varepsilon_{i}}\right)$ in $\Omega \times\left[0, T_{0}\right]$ such that $u^{\varepsilon_{i}} \in L^{\infty}\left(\left[0, T_{0}\right] ; V^{0,2}\right) \cap L^{p}\left(\left[0, T_{0}\right] ; V^{1, p}\right)$, $\left|\varphi^{\varepsilon_{i}}\right| \leqslant 1, \varphi^{\varepsilon_{i}} \in L^{\infty}\left(\left[0, T_{0}\right] ; C^{3}(\Omega)\right)$ and $\frac{\partial \varphi^{\varepsilon_{i}}}{\partial t} \in L^{\infty}\left(\left[0, T_{0}\right] ; C^{1}(\Omega)\right)$.

Proof. Assume that we are given a function $u(x, t)=\sum_{j=1}^{i} c_{j}^{\varepsilon_{i}}(t) \omega_{j}(x) \in C^{1 / 2}\left([0, T] ; V^{s, 2}\right)$ with

$$
c_{j}^{\varepsilon_{i}}(0)=\left(u_{0}, \omega_{j}\right), \quad \max _{t \in[0, T]}\left(\frac{1}{2} \sum_{j=1}^{i}\left|c_{j}^{\varepsilon_{i}}(t)\right|^{2}\right)^{1 / 2}+\sup _{0 \leqslant t_{1}<t_{2} \leqslant T} \sum_{j=1}^{i} \frac{\left|c_{j}^{\varepsilon_{i}}\left(t_{1}\right)-c_{j}^{\varepsilon_{i}}\left(t_{2}\right)\right|}{\left|t_{1}-t_{2}\right|^{1 / 2}} \leqslant \sqrt{2 E_{0}} .
$$

We let $\varphi(x, t)$ be the solution of the following parabolic equation:

$$
\frac{\partial \varphi}{\partial t}+\left(u * \zeta^{\varepsilon_{i}}\right) \cdot \nabla \varphi=\Delta \varphi-\frac{W^{\prime}(\varphi)}{\varepsilon_{i}^{2}}, \quad \varphi(x, 0)=\varphi_{0}^{\varepsilon_{i}}(x)
$$

The existence of such $\varphi$ with $|\varphi| \leqslant 1$ is guaranteed by the standard theory of parabolic equations ( [27]). By (3.11) and the Cauchy-Schwarz inequality, we can estimate

$$
\frac{d}{d t} \int_{\Omega}\left(\frac{\varepsilon_{i}|\nabla \varphi|^{2}}{2}+\frac{W(\varphi)}{\varepsilon_{i}}\right) d x \leqslant-\frac{\varepsilon_{i}}{2} \int_{\Omega}\left(\Delta \varphi-\frac{W^{\prime}(\varphi)}{\varepsilon_{i}^{2}}\right)^{2} d x+\frac{\varepsilon_{i}}{2} \int_{\Omega}\left\{\left(u * \zeta^{\varepsilon_{i}}\right) \cdot \nabla \varphi\right\}^{2} d x
$$


Since for any $t \in[0, T]$

$$
\begin{gathered}
\left\|u * \zeta^{\varepsilon_{i}}\right\|_{L^{\infty}(\Omega)}^{2} \leqslant \varepsilon_{i}^{-2 \gamma}\|u\|_{L^{\infty}(\Omega)}^{2} \leqslant \varepsilon_{i}^{-2 \gamma} \max _{1 \leqslant j \leqslant i}\left\|\omega_{j}(x)\right\|_{L^{\infty}(\Omega)}^{2} \sum_{j=1}^{i}\left|c_{j}^{\varepsilon_{i}}(t)\right|^{2} \leqslant c(i) E_{0} \\
\frac{d}{d t} \int_{\Omega}\left(\frac{\varepsilon_{i}|\nabla \varphi|^{2}}{2}+\frac{W(\varphi)}{\varepsilon_{i}}\right) d x \leqslant c(i) E_{0} \int_{\Omega} \frac{\varepsilon_{i}|\nabla \varphi|^{2}}{2} d x .
\end{gathered}
$$

This gives

$$
\sup _{0 \leqslant t \leqslant T} \frac{1}{\sigma} \int_{\Omega}\left(\frac{\varepsilon_{i}|\nabla \varphi|^{2}}{2}+\frac{W(\varphi)}{\varepsilon_{i}}\right) d x \leqslant e^{c(i) E_{0} T} E_{0} .
$$

Hence as long as $T \leqslant 1$,

$$
\left|D_{j}^{\varepsilon_{i}}(t)\right| \leqslant c\left\|\nabla \omega_{j}\right\|_{L^{\infty}(\Omega)} \frac{1}{\sigma} \int_{\Omega} \int_{\Omega} \varepsilon_{i}|\nabla \varphi(y)|^{2} \zeta^{\varepsilon_{i}}(x-y) d y d x \leqslant c(i) e^{c(i) E_{0}} E_{0}
$$

by $\nabla \omega_{j} \in L^{\infty}(\Omega)^{d^{2}}$ and (3.12).

Next we substitute the above solution $\varphi$ into the place of $\varphi^{\varepsilon_{i}}$, and solve (3.8) with the initial condition $c_{j}^{\varepsilon_{i}}(0)=\left(u_{0}, \omega_{j}\right)$. Since $\tau$ is locally Lipschitz with respect to $e(u)$, there is at least some short time $T_{1}$ such that (3.8) has a unique solution $\tilde{c}_{j}^{\varepsilon_{i}}(t)$ on $\left[0, T_{1}\right]$ with the initial condition $\tilde{c}_{j}^{\varepsilon_{i}}(0)=\left(u_{0}, \omega_{j}\right)$ for $1 \leqslant j \leqslant i$. We show that the solution exists up to $T_{0}=T_{0}\left(i, E_{0}, p, v_{0}\right)$ satisfying (3.10). Let $\tilde{c}(t)=\frac{1}{2} \sum_{j=1}^{i}\left|\tilde{c}_{j}^{\varepsilon_{i}}(t)\right|^{2}$. Then,

$$
\frac{d}{d t} \tilde{c}(t)=A_{j}^{\varepsilon_{i}} \tilde{c}_{j}^{\varepsilon_{i}}+B_{k l j} \tilde{c}_{k}^{\varepsilon_{i}} \tilde{c}_{l}^{\varepsilon_{i}} \tilde{c}_{j}^{\varepsilon_{i}}+D_{j}^{\varepsilon_{i}} \tilde{c}_{j}^{\varepsilon_{i}}
$$

By (1.1) $A_{j}^{\varepsilon_{i}} \tilde{c}_{j}^{\varepsilon_{i}} \leqslant 0$ hence

Therefore,

$$
\frac{d}{d t} \tilde{c}(t) \leqslant c\left(i, E_{0}\right)\left(\tilde{c}^{3 / 2}+\tilde{c}^{1 / 2}\right)
$$

$$
\arctan \sqrt{\tilde{c}(t)} \leqslant \arctan \sqrt{E_{0}}+2 c\left(i, E_{0}\right) t .
$$

We can also estimate $\left|d c_{j}^{\varepsilon_{i}} / d t\right|$ due to (3.8), (3.13), (3.14) and (1.2) depending only on $E_{0}, i, p, v_{0}$. Thus, by choosing $T_{0}$ small depending only on $E_{0}, i, p, v_{0}$ we have the existence of solution for $t \in\left[0, T_{0}\right]$ satisfying (3.10). We then prove the existence of a weak solution on $\Omega \times\left[0, T_{0}\right]$ by using Leray-Schauder fixed point theorem (see [27]). We define

$$
\tilde{u}(x, t)=\sum_{j=1}^{i} \tilde{c}_{j}^{\varepsilon_{i}}(t) \omega_{j}(x)
$$

and we define a map $\mathcal{L}: u \mapsto \tilde{u}$ as in the above procedure. Let

$$
\begin{aligned}
V\left(T_{0}\right) & :=\left\{u(x, t)=\sum_{j=1}^{i} c_{j}(t) \omega_{j}(x) ; \max _{t \in\left[0, T_{0}\right]}\left(\frac{1}{2} \sum_{j=1}^{i}\left|c_{j}(t)\right|^{2}\right)^{1 / 2}\right. \\
& \left.+\sup _{0 \leqslant t_{1}<t_{2} \leqslant T_{0}} \sum_{j=1}^{i} \frac{\left|c_{j}\left(t_{1}\right)-c_{j}\left(t_{2}\right)\right|}{\left|t_{1}-t_{2}\right|^{1 / 2}} \leqslant \sqrt{2 E_{0}}, c_{j}(0)=\left(u_{0}, \omega_{j}\right), c_{j} \in C^{1 / 2}\left(\left[0, T_{0}\right]\right)\right\} .
\end{aligned}
$$


Then $V\left(T_{0}\right)$ is a closed, convex subset of $C^{1 / 2}\left(\left[0, T_{0}\right] ; V_{i}^{0,2}\right)$ equipped with the norm

$$
\|u\|_{V\left(T_{0}\right)}=\max _{t \in\left[0, T_{0}\right]}\left(\frac{1}{2} \sum_{j=1}^{i}\left|c_{j}(t)\right|^{2}\right)^{1 / 2}+\sup _{0 \leqslant t_{1}<t_{2} \leqslant T_{0}} \sum_{j=1}^{i} \frac{\left|c_{j}\left(t_{1}\right)-c_{j}\left(t_{2}\right)\right|}{\left|t_{1}-t_{2}\right|^{1 / 2}}
$$

and by the above argument $\mathcal{L}: V\left(T_{0}\right) \rightarrow V\left(T_{0}\right)$. Moreover the image of $\mathcal{L}$ is bounded in $C^{0,1}\left(\left[0, T_{0}\right] ; V_{i}^{0,2}\right)$ due to the estimate on $\left|d c_{j}^{\varepsilon_{i}} / d t\right|$. Thus by the Ascoli-Arzelà compactness theorem $\mathcal{L}$ is a compact operator. Therefore by using the Leray-Schauder fixed point theorem $\AA \mathrm{C} \mathcal{L}$ has a fixed point $u^{\varepsilon_{i}} \in V\left(T_{0}\right)$. We denote by $\varphi^{\varepsilon_{i}}$ the solution of (3.6) and (3.7). Then $\left(u^{\varepsilon_{i}}, \varphi^{\varepsilon_{i}}\right)$ is a weak solution of (3.4)-(3.7) in $\Omega \times\left[0, T_{0}\right]$. Note that we have the required regularities for $\varphi^{\varepsilon_{i}}$ due to the regularity of $u^{\varepsilon_{i}} * \zeta^{\varepsilon_{i}}$ in $x$ and by the standard parabolic regularity theory.

THEOREM 3.3 Let $\left(u^{\varepsilon_{i}}, \varphi^{\varepsilon_{i}}\right)$ be the weak solution of (3.4)-(3.7) with (3.9) in $\Omega \times[0, T]$. Then the following energy estimate holds:

$$
\begin{aligned}
\int_{\Omega} \frac{1}{\sigma}\left(\frac{\varepsilon_{i}\left|\nabla \varphi^{\varepsilon_{i}}(\cdot, T)\right|^{2}}{2}\right. & \left.+\frac{W\left(\varphi^{\varepsilon_{i}}(\cdot, T)\right)}{\varepsilon_{i}}\right)+\frac{\left|u^{\varepsilon_{i}}(\cdot, T)\right|^{2}}{2} d x \\
& +\int_{0}^{T} \int_{\Omega} \frac{\varepsilon_{i}}{\sigma}\left(\Delta \varphi^{\varepsilon_{i}}-\frac{W^{\prime}\left(\varphi^{\varepsilon_{i}}\right)}{\varepsilon_{i}^{2}}\right)^{2}+v_{0}\left|e\left(u^{\varepsilon_{i}}\right)\right|^{p} d x d t \leqslant E_{0}+o(1)
\end{aligned}
$$

Moreover for any $0 \leqslant T_{1}<T_{2}<\infty$

$$
\int_{T_{1}}^{T_{2}}\left\|u^{\varepsilon_{i}}(\cdot, t)\right\|_{W^{1, p}(\Omega)}^{p} d t \leqslant c_{K}\left\{v_{0}^{-1} E_{0}+\left(T_{2}-T_{1}\right) E_{0}^{\frac{p}{2}}\right\}+o(1) .
$$

Proof. Since $\left(u^{\varepsilon_{i}}, \varphi^{\varepsilon_{i}}\right)$ is the weak solution of (3.4)-(3.7), we derive

$$
\begin{aligned}
\frac{d}{d t} \int_{\Omega} & \frac{1}{\sigma}\left(\frac{\varepsilon_{i}\left|\nabla \varphi^{\varepsilon_{i}}\right|^{2}}{2}+\frac{W\left(\varphi^{\varepsilon_{i}}\right)}{\varepsilon_{i}}\right)+\frac{\left|u^{\varepsilon_{i}}\right|^{2}}{2} d x \\
= & \int_{\Omega}-\frac{\varepsilon_{i}}{\sigma} \frac{\partial \varphi^{\varepsilon_{i}}}{\partial t}\left(\Delta \varphi^{\varepsilon_{i}}-\frac{W^{\prime}\left(\varphi^{\varepsilon_{i}}\right)}{\varepsilon_{i}^{2}}\right)+\frac{\partial u^{\varepsilon_{i}}}{\partial t} \cdot u^{\varepsilon_{i}} d x \\
= & \int_{\Omega}-\frac{\varepsilon_{i}}{\sigma}\left(\Delta \varphi^{\varepsilon_{i}}-\frac{W^{\prime}\left(\varphi^{\varepsilon_{i}}\right)}{\varepsilon_{i}^{2}}-\left(u^{\varepsilon_{i}} * \zeta^{\varepsilon_{i}}\right) \cdot \nabla \varphi^{\varepsilon_{i}}\right)\left(\Delta \varphi^{\varepsilon_{i}}-\frac{W^{\prime}\left(\varphi^{\varepsilon_{i}}\right)}{\varepsilon^{2}}\right) d x \\
& \quad+\int_{\Omega}\left\{\operatorname{div} \tau\left(\varphi^{\varepsilon_{i}}, e\left(u^{\varepsilon_{i}}\right)\right)-u^{\varepsilon_{i}} \cdot \nabla u^{\varepsilon_{i}}-\frac{\varepsilon_{i}}{\sigma} \operatorname{div}\left(\left(\nabla \varphi^{\varepsilon_{i}} \otimes \nabla \varphi^{\varepsilon_{i}}\right) * \zeta^{\varepsilon_{i}}\right)\right\} \cdot u^{\varepsilon_{i}} d x \\
= & I_{1}+I_{2} .
\end{aligned}
$$

Since $\operatorname{div}\left(u^{\varepsilon_{i}} * \zeta^{\varepsilon_{i}}\right)=\left(\operatorname{div} u^{\varepsilon_{i}}\right) * \zeta^{\varepsilon_{i}}=0$,

$$
\sigma I_{1}=-\int_{\Omega} \varepsilon_{i}\left(\Delta \varphi^{\varepsilon_{i}}-\frac{W^{\prime}(\varphi)}{\varepsilon_{i}^{2}}\right)^{2} d x+\varepsilon_{i} \int_{\Omega}\left(u^{\varepsilon_{i}} * \zeta^{\varepsilon_{i}}\right) \cdot \nabla \varphi^{\varepsilon_{i}} \Delta \varphi^{\varepsilon_{i}} d x
$$

For $I_{2}$, with (1.1)

$$
\int_{\Omega} \operatorname{div} \tau\left(\varphi^{\varepsilon_{i}}, e\left(u^{\varepsilon_{i}}\right)\right) \cdot u^{\varepsilon_{i}} d x=-\int_{\Omega} \tau\left(\varphi^{\varepsilon_{i}}, e\left(u^{\varepsilon_{i}}\right)\right): e\left(u^{\varepsilon_{i}}\right) d x \leqslant-v_{0} \int_{\Omega}\left|e\left(u^{\varepsilon_{i}}\right)\right|^{p} d x .
$$


Moreover, the second term of $I_{2}$ vanishes by $\operatorname{div} u^{\varepsilon_{i}}=0$ and

$$
\begin{aligned}
-\int_{\Omega} \varepsilon_{i} \operatorname{div}\left(\nabla \varphi^{\varepsilon_{i}} \otimes \nabla \varphi^{\varepsilon_{i}} * \zeta^{\varepsilon_{i}}\right) \cdot u^{\varepsilon_{i}} d x & =-\int_{\Omega} \varepsilon_{i}\left(\nabla \frac{\left|\nabla \varphi^{\varepsilon_{i}}\right|^{2}}{2}+\nabla \varphi^{\varepsilon_{i}} \Delta \varphi^{\varepsilon_{i}}\right) * \zeta^{\varepsilon} \cdot u^{\varepsilon_{i}} d x \\
& =-\varepsilon_{i} \int_{\Omega}\left(u^{\varepsilon_{i}} * \zeta^{\varepsilon_{i}}\right) \cdot \nabla \varphi^{\varepsilon_{i}} \Delta \varphi^{\varepsilon_{i}} d x .
\end{aligned}
$$

Hence (3.17) becomes

$\frac{d}{d t} \int_{\Omega} \frac{1}{\sigma}\left(\frac{\varepsilon_{i}\left|\nabla \varphi^{\varepsilon_{i}}\right|^{2}}{2}+\frac{W\left(\varphi^{\varepsilon_{i}}\right)}{\varepsilon_{i}}\right)+\frac{\left|u^{\varepsilon_{i}}\right|^{2}}{2} d x \leqslant-\int_{\Omega} \frac{\varepsilon_{i}}{\sigma}\left(\Delta \varphi^{\varepsilon_{i}}-\frac{W^{\prime}\left(\varphi^{\varepsilon_{i}}\right)}{\varepsilon_{i}^{2}}\right)^{2}+v_{0}\left|e\left(u^{\varepsilon_{i}}\right)\right|^{p} d x$.

Integrating with respect to $t$ over $t \in[0, T]$ and by (3.9), we obtain (3.15). The proof of (3.16) follows from (3.15) and Theorem 2.7.

Proof of Theorem 3.1. For each fixed $i$ we have a short time existence for $\left[0, T_{0}\right]$ where $T_{0}$ depends only on $i, E_{0}, p, \nu_{0}$ at $t=0$. By Theorem 3.3 the energy at $t=T_{0}$ is again bounded by $E_{0}+o(1)$. By repeatedly using Lemma 3.2, Theorem 3.1 follows.

\section{Proof of main theorem}

In this section we first prove that $\left\{\varphi^{\varepsilon_{i}}\right\}_{i=1}^{\infty}$ in Section 3 and the associated surface energy measures $\left\{\mu_{t}^{\varepsilon_{i}}\right\}_{i=1}^{\infty}$ converge subsequentially to $\varphi$ and $\mu_{t}$ which satisfy the properties described in Theorem 2.3. Most of the technical and essential ingredients have been proved in [30] and we only need to check the conditions to apply the results. We then prove that the limit velocity field satisfies the weak non-Newtonian flow equation, concluding the proof of Theorem 2.3.

First we recall the upper density ratio bound of the surface energy.

Theorem 4.1 ( [30, Theorem 3.1]) Suppose $d \geqslant 2, \Omega=\mathbb{T}^{d}, p>\frac{d+2}{2}, \frac{1}{2}>\gamma \geqslant 0,1 \geqslant \varepsilon>0$ and $\varphi$ satisfies

$$
\begin{aligned}
\frac{\partial \varphi}{\partial t}+u \cdot \nabla \varphi=\Delta \varphi-\frac{W^{\prime}(\varphi)}{\varepsilon^{2}} & \text { on } \Omega \times[0, T], \\
\varphi(x, 0)=\varphi_{0}(x) & \text { on } \Omega,
\end{aligned}
$$

where $\nabla^{i} u, \nabla^{j} \varphi, \nabla^{k} \varphi_{t} \in C(\Omega \times[0, T])$ for $0 \leqslant i, k \leqslant 1$ and $0 \leqslant j \leqslant 3$. Let $\mu_{t}$ be the Radon measure on $\Omega$ defined by

$$
\int_{\Omega} \phi(x) d \mu_{t}(x)=\frac{1}{\sigma} \int_{\Omega} \phi(x)\left(\frac{\varepsilon|\nabla \varphi(x, t)|^{2}}{2}+\frac{W(\varphi(x, t))}{\varepsilon}\right) d x
$$


for $\phi \in C(\Omega)$, where $\sigma=\int_{-1}^{1} \sqrt{2 W(s)} d s$. We assume also that

$$
\begin{gathered}
\sup _{\Omega}\left|\varphi_{0}\right| \leqslant 1 \text { and } \sup _{\Omega} \varepsilon^{i}\left|\nabla^{i} \varphi_{0}\right| \leqslant c_{1} \text { for } 1 \leqslant i \leqslant 3, \\
\sup _{\Omega}\left(\frac{\varepsilon\left|\nabla \varphi_{0}\right|^{2}}{2}-\frac{W\left(\varphi_{0}\right)}{\varepsilon}\right) \leqslant \varepsilon^{-\gamma}, \\
\sup _{\Omega \times[0, T]}\left\{\varepsilon^{\gamma}|u|, \varepsilon^{1+\gamma}|\nabla u|\right\} \leqslant c_{2}, \\
\int_{0}^{T}\|u(\cdot, t)\|_{W^{1, p}(\Omega)}^{p} d t \leqslant c_{3} .
\end{gathered}
$$

Define for $t \in[0, T]$

$$
D(t)=\max \left\{\sup _{x \in \Omega, 0<r \leqslant \frac{1}{2}} \frac{1}{\omega_{d-1} r^{d-1}} \mu_{t}\left(B_{r}(x)\right), 1\right\}, \quad D(0) \leqslant D_{0} .
$$

Then there exist $\epsilon_{1}>0$ which depends only on $d, p, W, c_{1}, c_{2}, c_{3}, D_{0}, \gamma$ and $T$, and $c_{4}$ which depends only on $c_{3}, d, p, D_{0}$ and $T$ such that for all $0<\varepsilon \leqslant \epsilon_{1}$,

$$
\sup _{0 \leqslant t \leqslant T} D(t) \leqslant c_{4}
$$

Using this we prove

Proposition 4.2 For $\left\{\varphi^{\varepsilon_{i}}\right\}_{i=1}^{\infty}$ in Theorem 3.1, define $\mu_{t}^{\varepsilon_{i}}$ as in (4.3) replacing $\varphi$ by $\varphi^{\varepsilon_{i}}$, and define $D^{\varepsilon_{i}}(t)$ as in (4.8) replacing $\mu_{t}$ by $\mu_{t}^{\varepsilon_{i}}$. Given $0<T<\infty$, there exists $c_{5}$ which depends only on $E_{0}, v_{0}, \gamma, D_{0}, T, d, p$ and $W$ such that

$$
\sup _{0 \leqslant t \leqslant T} D^{\varepsilon_{i}}(t) \leqslant c_{5}
$$

for all sufficiently large $i$.

Proof. We only need to check the conditions of Theorem 4.1 for $\varphi^{\varepsilon_{i}}$ and $\mu_{t}^{\varepsilon_{i}}$. Note that $u$ in (4.1) is replaced by $u^{\varepsilon_{i}} * \zeta^{\varepsilon_{i}}$. We have $d \geqslant 2, \Omega=\mathbb{T}^{d}, p>\frac{d+2}{2}, \frac{1}{2}>\gamma \geqslant 0,1 \geqslant \varepsilon>0$ and (4.1) and (4.2). The regularity of functions is guaranteed in Theorem 3.1. With an appropriate choice of $c_{1}$, (4.4) is satisfied for all sufficiently large $i$ due to the choice of $\varepsilon_{i}$ in (3.2). The sup bound (4.5) is satisfied with even 0 on the right-hand side instead of $\varepsilon_{i}^{-\gamma}$. The bound for $u^{\varepsilon_{i}} * \zeta^{\varepsilon_{i}}$ (4.6) is satisfied due to (3.1) and (3.15), and (4.7) is satisfied due to (3.16). Thus we have all the conditions, and Theorem 4.1 proves the claim.

We next prove

PROPOSITION 4.3 For $\left\{u^{\varepsilon_{i}} * \zeta^{\varepsilon_{i}}\right\}_{i=1}^{\infty}$ in Theorem 3.1, there exist a subsequence (denoted by the same index) and a limit $u \in L^{\infty}\left([0, \infty) ; V^{0,2}\right) \cap L_{l o c}^{p}\left([0, \infty) ; V^{1, p}\right)$ such that for any $0<T<\infty$ $u^{\varepsilon_{i}} * \zeta^{\varepsilon_{i}} \rightarrow u$ weakly in $L^{p}\left([0, T] ; W^{1, p}(\Omega)^{d}\right), \quad u^{\varepsilon_{i}} * \zeta^{\varepsilon_{i}} \rightarrow u$ strongly in $L^{2}\left([0, T] ; L^{2}(\Omega)^{d}\right)$. 
Proof. Let $\psi \in V^{s, 2}$ with $\|\psi\|_{V^{s, 2}} \leqslant 1$. With (3.4), (3.5) and integration by parts, we have

$$
\begin{aligned}
\left(\frac{\partial u^{\varepsilon_{i}}}{\partial t}, \psi\right) & =\left(\frac{\partial u^{\varepsilon_{i}}}{\partial t}, P_{i} \psi\right) \\
& =\left(-u^{\varepsilon_{i}} \cdot \nabla u^{\varepsilon_{i}}+\operatorname{div} \tau\left(\varphi^{\varepsilon_{i}}, e\left(u^{\varepsilon_{i}}\right)\right)-\frac{\varepsilon_{i}}{\sigma} \operatorname{div}\left(\nabla \varphi^{\varepsilon_{i}} \otimes \nabla \varphi^{\varepsilon_{i}}\right) * \zeta^{\varepsilon_{i}}, P_{i} \psi\right) \\
& =\left(u^{\varepsilon_{i}} \otimes u^{\varepsilon_{i}}-\tau\left(\varphi^{\varepsilon_{i}}, e\left(u^{\varepsilon_{i}}\right)\right)+\frac{\varepsilon_{i}}{\sigma}\left(\nabla \varphi^{\varepsilon_{i}} \otimes \nabla \varphi^{\varepsilon_{i}}\right) * \zeta^{\varepsilon_{i}}, \nabla P_{i} \psi\right) .
\end{aligned}
$$

Here we remark that

$$
\left\|\nabla P_{i} \psi\right\|_{L^{\infty}(\Omega)} \leqslant c(d)\left\|P_{i} \psi\right\|_{W^{s, 2}(\Omega)} \leqslant c(d)\|\psi\|_{W^{s, 2}(\Omega)}=c(d)\|\psi\|_{V^{s, 2}} \leqslant c(d)
$$

by $s>\frac{d+2}{2}$ and properties of $P_{i}$ (see [29] or [33, p.290]). Thus by (1.2) and (3.15), we obtain

$$
\left(\frac{\partial u^{\varepsilon_{i}}}{\partial t}, \psi\right) \leqslant c\left(d, p, v_{0}\right)\left(1+E_{0}+\left\|u^{\varepsilon_{i}}\right\|_{W^{1, p}(\Omega)}^{p-1}\right) .
$$

Again using (3.15) and integrating in time we obtain

$$
\int_{0}^{T}\left\|\frac{\partial u^{\varepsilon_{i}}}{\partial t}\right\|_{\left(V^{s, 2}\right)^{*}}^{\frac{p}{p-1}} d t \leqslant c\left(d, p, E_{0}, v_{0}, T\right) .
$$

Now we use Aubin-Lions compactness Theorem [29, p.57] with $B_{0}=V^{s, 2}, B=V^{0,2} \subset L^{2}(\Omega)^{d}$, $B_{1}=\left(V^{s, 2}\right)^{*}, p_{0}=p$ and $p_{1}=\frac{p}{p-1}$. Then there exists a subsequence still denoted by $\left\{u^{\varepsilon_{i}}\right\}_{i=1}^{\infty}$ such that

$$
u^{\varepsilon_{i}} \rightarrow u \quad \text { in } L^{p}\left([0, T] ; L^{2}(\Omega)^{d}\right) .
$$

Since $p>2$, the strong convergence also holds in $L^{2}\left([0, T] ; L^{2}(\Omega)^{d}\right)$. Note that we also have proper norm bounds to extract weakly convergent subsequences due to (3.15). For each $T_{n}$ which diverges to $\infty$ as $n \rightarrow \infty$, we choose a subsequence and by choosing a diagonal subsequence, we obtain the convergent subsequence with (4.11) with $u^{\varepsilon_{i}}$ instead of $u^{\varepsilon_{i}} * \zeta^{\varepsilon_{i}}$. It is not difficult to show at this point that the same convergence results hold for $u^{\varepsilon_{i}} * \zeta^{\varepsilon_{i}}$ as in (4.11).

Proof of main theorem. At this point, the rest of the proof concerning the existence of the limit Radon measure $\mu_{t}$ and the limit $\varphi=\lim _{i \rightarrow \infty} \varphi^{\varepsilon_{i}}$ and their respective properties described in Theorem 2.3 can be proved by almost line by line identical argument in [30, Section 4, 5]. The only difference is that the energy $E_{0}$ in [30] depends also on $T$, while in this paper $E_{0}$ depends only on the initial data due to (3.15). This allows us to have time-global estimates such as $u \in L^{\infty}\left([0, \infty) ; V^{0,2}\right)$ and $\sup _{t \in[0, \infty)}\|\varphi\|_{B V(\Omega)}<\infty$. The argument in [30] then complete the existence proof of Theorem 2.3 (b), (c) along with (iii)-(vi). We still need to prove (a), (i) and (ii).

Due to (4.12), (1.2) and (3.16) we may extract a further subsequence so that

$$
\begin{aligned}
& \frac{\partial u^{\varepsilon_{i}}}{\partial t} \rightarrow \frac{\partial u}{\partial t} \text { weakly in } L^{\frac{p}{p-1}}\left([0, T] ;\left(V^{s, 2}\right)^{*}\right), \\
& \tau\left(\varphi^{\varepsilon_{i}}, e\left(u^{\varepsilon_{i}}\right)\right) \rightarrow \hat{\tau} \text { weakly in } L^{\frac{p}{p-1}}\left([0, T] ; L^{\frac{p}{p-1}}(\Omega)^{d^{2}}\right) .
\end{aligned}
$$

For $\omega_{j} \in V^{s, 2}(j=1, \cdots)$ and $h \in C_{c}^{\infty}((0, T))$ we have

$$
\int_{\Omega} \operatorname{div}\left(\left(\nabla \varphi^{\varepsilon_{i}} \otimes \nabla \varphi^{\varepsilon_{i}}\right) * \zeta^{\varepsilon_{i}}\right) \cdot h \omega_{j} d x=\int_{\Omega}\left(\Delta \varphi^{\varepsilon_{i}}-\frac{W^{\prime}\left(\varphi^{\varepsilon_{i}}\right)}{\varepsilon_{i}^{2}}\right) \nabla \varphi^{\varepsilon_{i}} \cdot h \omega_{j} * \zeta^{\varepsilon_{i}} d x
$$


by integration by parts and $\operatorname{div} \omega_{j}=0$. Thus the argument in [29, p. 212] and the similar convergence argument in [30] show

$$
\int_{0}^{T}\left\{\left(\frac{\partial u}{\partial t}, h \omega_{j}\right)+\int_{\Omega}(u \cdot \nabla u) \cdot h \omega_{j}+h \hat{\tau}: e\left(\omega_{j}\right) d x\right\} d t=\int_{0}^{T} \int_{\Omega} H \cdot h \omega_{j} d \mu_{t} d t
$$

Again by the similar argument using the density ratio bound and Theorem 2.1 one shows by the density argument and (4.14) that $\frac{\partial u}{\partial t} \in L^{\frac{p}{p-1}}\left([0, T] ;\left(V^{1, p}\right)^{*}\right)$ and

$$
\int_{0}^{T}\left\{\left(\frac{\partial u}{\partial t}, v\right)+\int_{\Omega}(u \cdot \nabla u) \cdot v+\hat{\tau}: e(v) d x\right\} d t=\int_{0}^{T} \int_{\Omega} H \cdot v d \mu_{t} d t .
$$

for all $v \in L^{p}\left([0, T] ; V^{1, p}\right)$. We next prove

$$
\int_{0}^{T} \int_{\Omega} \hat{\tau}: e(v) d x d t=\int_{0}^{T} \int_{\Omega} \tau(\varphi, e(u)): e(v) d x d t
$$

for all $v \in C_{c}^{\infty}((0, T) ; v)$. As in [29, p. $\left.213(5.43)\right]$, we may deduce that

$$
\frac{1}{2}\left\|u\left(t_{1}\right)\right\|_{L^{2}(\Omega)}^{2}+\int_{0}^{t_{1}} \int_{\Omega} \hat{\tau}: e(u) d x d t \geqslant \int_{0}^{t_{1}} \int_{\Omega} H \cdot u d \mu_{t} d t+\frac{1}{2}\|u(0)\|_{L^{2}(\Omega)}^{2}
$$

for a.e. $t_{1} \in[0, T]$. We set for any $v \in V^{1, p}$

$$
A_{i}^{t_{1}}=\int_{0}^{t_{1}} \int_{\Omega}\left(\tau\left(\varphi^{\varepsilon_{i}}, e\left(u^{\varepsilon_{i}}\right)\right)-\tau\left(\varphi^{\varepsilon_{i}}, e(v)\right):\left(e\left(u^{\varepsilon_{i}}\right)-e(v)\right) d x d t+\frac{1}{2}\left\|u^{\varepsilon_{i}}\left(t_{1}\right)\right\|_{L^{2}(\Omega)}^{2} .\right.
$$

The property (1.3) of $e(\cdot)$ shows that the first term of (4.18) is non-negative. We may further assume that $u^{\varepsilon_{i}}\left(t_{1}\right)$ converges weakly to $u\left(t_{1}\right)$ in $L^{2}(\Omega)^{d}$ thus we have

$$
\liminf _{i \rightarrow \infty} A_{i}^{t_{1}} \geqslant \frac{1}{2}\left\|u\left(t_{1}\right)\right\|_{L^{2}(\Omega)}^{2}
$$

By (3.4) we have

$$
\begin{aligned}
A_{i}^{t_{1}}=\frac{1}{2}\left\|u^{\varepsilon_{i}}(0)\right\|_{L^{2}(\Omega)}^{2} & -\frac{\varepsilon_{i}}{\sigma} \int_{0}^{t_{1}} \int_{\Omega} \operatorname{div}\left(\left(\nabla \varphi^{\varepsilon_{i}} \otimes \nabla \varphi^{\varepsilon_{i}}\right) * \zeta^{\varepsilon_{i}}\right) \cdot u^{\varepsilon_{i}} \\
& -\int_{0}^{t_{1}} \int_{\Omega} \tau\left(\varphi^{\varepsilon_{i}}, e\left(u^{\varepsilon_{i}}\right)\right): e(v)+\tau\left(\varphi^{\varepsilon_{i}}, e(v)\right):\left(e\left(u^{\varepsilon_{i}}\right)-e(v)\right) d x d t
\end{aligned}
$$

which converges to

$$
\begin{aligned}
A^{t_{1}}=\frac{1}{2}\|u(0)\|_{L^{2}(\Omega)}^{2}+\int_{0}^{t_{1}} \int_{\Omega} H \cdot u d \mu_{t} d t & \\
& \quad-\int_{0}^{t_{1}} \int_{\Omega} \hat{\tau}: e(v)+\tau(\varphi, e(v)):(e(u)-e(v)) d x d t .
\end{aligned}
$$


The convergence of the second term is discussed in [30, Proposition 4.5] and we used that $\varphi^{\varepsilon_{i}}$ converges to $\varphi$ a.e. on $\Omega \times[0, T]$. By (4.17), (4.19) and (4.20), we deduce that

$$
\int_{0}^{t_{1}} \int_{\Omega}(\hat{\tau}-\tau(\varphi, e(v))):(e(u)-e(v)) d x d t \geqslant 0
$$

By choosing $v=u+\epsilon \tilde{v}$, divide by $\epsilon$ and letting $\epsilon \rightarrow 0$, we prove (4.16). Finally, (2.7) follows from (4.16), the strong $L^{1}(\Omega \times[0, T])$ convergence of $\varphi^{\varepsilon_{i}}$, the lower semicontinuity of the mean curvature square term (see [30, Proposition 4.4]) and the energy equality appearing in Theorem 3.3. This concludes the proof of Theorem 2.3.

\section{REFERENCES}

1. AbeLs, H., The initial value problem for the Navier-Stokes equations with a free surface in $L^{q}$-Sobolev spaces, Adv. Diff. Eqns. 10 (2005), 45-64. Zbl1105.35072 MR2106120

2. Abels, H., On generalized solutions of two-phase flows for viscous incompressible fluids, Interface. Free Bound. 9 (2007), 31-65. Zbl1124.35060 MR2317298

3. AbELs, H., On a diffuse interface model for two-phase flows of viscous, incompressible fluids with matched densities, Arch. Ration. Mech. Anal. 194 (2009), 463-506. Zblpre05640833 MR2563636

4. Abels, H., Longtime behavior of solutions of a Navier-Stokes/Cahn-Hilliard system, Nonlocal and abstract parabolic equations and their applications, 9-19, Banach Center Publ. 86, Polish Acad. Sci. Inst. Math., Warsaw (2009). Zb11167.76008 MR2571478

5. AbELs, H. \& RÖGER, M., Existence of weak solutions for a non-classical sharp interface model for a two-phase flow of viscous, incompressible fluids, Ann. Inst. H. Poincaré Anal. Non Linéaire 26 (2009), 2403-2424. Zbl1181.35343 MR2569901

6. Allard, W., On the first variation of a varifold, Ann. of Math. 95 (1972), 417-491. Zb10252. 49028 MR0307015

7. Anderson, D. M., McFadden, G. B., \& Wheeler, A. A., Diffuse-interface methods in fluid mechanics, Annu. Rev. Fluid Mech. 30, Annual Reviews, Palo Alto, CA, (1998), 139-165. MR1609626

8. BeAle, J. T., The initial value problem for the Navier-Stokes equations with a free surface, Comm. Pure. Appl. Math. 34 (1981), 359-392. Zb10464.76028 MR0611750

9. BeAle, J. T., Large-time regularity of viscous surface waves, Arch. Ration. Mech. Anal. 84 (1984), 307352. Zbl0545.76029 MR0721189

10. BOYER, F., Mathematical study of multi-phase flow under shear through order parameter formulation, Asymptot. Anal. 20 (1999), 175-212. Zb10937. 35123 MR1700669

11. Boyer, F., Nonhomogeneous Cahn-Hilliard fluids. Ann. Inst. H. Poincaré Anal. Non Linéaire 18 (2001), 225-259. Zbl1037.76062 MR1808030

12. BOYER, F., A theoretical and numerical model for the study of incompressible mixture flows, Computers and Fluids 31 (2002), 41-68. Zbl 1057.76060

13. Boyer, F. \& FABrie, P., Persistency of 2D perturbations of one-dimensional solutions for a CahnHilliard flow model under high shear, Asymptotic Anal. 33 (2003), 107-151. Zbl1137. 76823 MR1977766

14. Brakke, K., The motion of a surface by its mean curvature, Princeton University Press, Princeton, NJ (1978). Zbl0386.53047 MR0485012

15. Chen, Y. \& Struwe, M., Existence and partial regularity for the solutions to evolution problems for harmonic maps, Math. Z. 201 (1989), 83-103. Zb10652. 58024 MR0990191

16. Evans, L. C., Partial differential equations, Graduate Studies in Math. AMS, (1998). Zb10902. 35002 MR1625845 
17. Evans, L. C. \& GARIEPy, R. F., Measure theory and fine properties of functions, Studies in Advanced Math. CRC Press (1992). Zb10804. 28001 MR1158660

18. Gal, C. G. \& Grasselli, M., Asymptotic behavior of a Cahn-Hilliard-Navier-Stokes system in 2D, Ann. Inst. H. Poincaré Anal. Non Linéaire 27 (2010), 401-436. Zbl1184. 35055 MR2580516

19. GAL, C. G. \& GRASSELli, M., Trajectory attractors for binary fluid mixtures in 3D, Chin. Ann. Math. Ser. B 31 (2010), 655-678. Zbl1223. 35079 MR2726061

20. Giga, Y. \& Takahashi, S., On global weak solutions of the nonstationary two phase Stokes flow, SIAM J. Math. Anal. 25 (1994), 876-893. Zb10806. 35137 MR1271315

21. Hohenberg, P. C. \& Halperin, B. I., Theory of dynamical critical phenomena, Rev. Modern Phys. 49 (1977) 435-479.

22. Huisken, G., Asymptotic behavior for singularities of the mean curvature flow, J. Diff. Geom. 31 (1990), 285-299. Zb10694.53005 MR1030675

23. Hutchinson,J. E. \& Tonegawa, Y., Convergence of phase interfaces in the van der Waals-Cahn-Hilliard theory, Calc. Var. PDE 10 (2000), 49-84. Zbl1070.49026 MR1803974

24. Ilmanen, T., Convergence of the Allen-Cahn equation to Brakke's motion by mean curvature, J. Diff. Geom. 38 (1993), 417-461. Zbl0784. 53035 MR1237490

25. IlmANEN, T., Elliptic regularization and partial regularity for motion by mean curvature, Mem. Amer. Math. Soc. 108 (1994). Zb10798.35066 MR1196160

26. Kim, N., CONSiglieri, L. \& Rodrigues, J. F., On non-Newtonian incompressible fluids with phase transitions, Math. Meth. Appl. Sci. 29 (2006), 1523-1541. Zb11101.76004 MR2249576

27. Ladyzhenskaya, O. A., Solonnikov, N. A., \& Uraltseva, N. N., Linear and Quasilinear Equations Of Parabolic Type, Transl. Math. Monographs, Vol. 23, Amer. Math. Soc. (1968). Zbl0174. 15403 MR0241822

28. Lin, F. H. \& LIU, C., Nonparabolic dissipative systems modeling the flow of liquid crystals, Comm. Pure. Appl. Math. 48 (1995), 501-537. Zb10842.35084 MR1329830

29. Lions, J. L., Quelques Méthodes de Résolution des Problèmes aux Limites Non Linéaires, Dunod, Paris (1969). Zb10189.40603 MR0259693

30. Liu, C., Sato, N., \& Tonegawa, Y., On the existence of mean curvature flow with transport term, Interface. Free Bound. 12 (2010), 251-277. Zbl1201. 53076 MR2652019

31. LiU, C. \& Walkington, N. J., An Eulerian description of fluids containing visco-hyperelastic particles, Arch. Ration. Mech. Anal. 159 (2001), 229-252. Zbl1009.76093 MR1857673

32. Maekawa, Y., On a free boundary problem for viscous incompressible flows, Interface. Free Bound. 9 (2007), 549-589. Zbl1132.76303 MR2358216

33. MÁlek, J., NeČAs, J., Rokyta, M., \& RŮŽIČKA, M., Weak and measure-valued solutions to evolutionary PDEs, Appl. Math. Math. Comput. 13, Chapman \& Hall, London (1996). Zb10851. 35002 MR1409366

34. Meyers, N. G. \& Ziemer, W. P., Integral inequalities of Poincaré and Wirtinger type for BV functions, Amer. J. Math. 99 (1977), 1345-1360. Zbl0416. 46025 MR0507433

35. Mugnai, L. \& RöGER, M., Convergence of perturbed Allen-Cahn equations to forced mean curvature flow, Indiana Univ. Math. J. 60 (2011), 41-76. Zblpre06039326

36. Nouri, A. \& Poupaud, F., An existence theorem for the multifluid Navier-Stokes Problem, J. Diff. Eqns. 122 (1995), 71-88. Zbl0842. 35079 MR1356130

37. Plotnikov, P. I., Generalized solutions to a free boundary problem of motion of a non-Newtonian fluid, Siberian Math. J. 34 (1993), 704-716. Zb10814. 76007 MR1248797

38. RöGer, M. \& SchÄtzle, R., On a modified conjecture of De Giorgi, Math. Z. 254 (2006), 675-714. Zbl1126.49010 MR2253464 
39. SAto, N., A simple proof of convergence of the Allen-Cahn equation to Brakke's motion by mean curvature, Indiana Univ. Math. J. 57 (2008), 1743-1751. Zbl1160. 35444 MR2440879

40. Simon, L., Lectures on geometric measure theory, Proc. Centre Math. Anal. Austral. Nat. Univ. 3 (1983). Zbl0546.49019 MR0756417

41. Solonnikov, V. A., Estimates of the solution of a certain initial-boundary value problem for a linear nonstationary system of Navier-Stokes equations, Zap. Nauchn. Sem. Leningrad. Otdel. Mat. Inst. Steklov. (LOMI) 59 (1976), 178-254, 257 (in Russian). MR0460931

42. Solonnikov, V. A., On the transient motion of an isolated volume of viscous incompressible fluid, Math. USSR-Izv. 31 (1988), 381-405. Zbl0850.76180

43. Soner, H. M., Convergence of the phase-field equations to the Mullins-Sekerka problem with kinetic undercooling, Arch. Ration. Mech. Anal. 131 (1995), 139-197. Zb10829.73010 MR1346368

44. TONEGAWA, Y., Integrality of varifolds in the singular limit of reaction-diffusion equations, Hiroshima Math. J. 33 (2003), 323-341. Zbl1059.35061 MR2040901

45. ZHAO, L., WU, H., \& HUANG, H., Convergence to equilibrium for a phase-field model for the mixture of two viscous incompressible fluids, Commun. Math. Sci. 7 (2009), 939-962. Zbl1183. 35224 MR2604626

46. ZIEMER, W. P., Weakly differentiable functions, Springer-Verlag (1989). Zb10692 . 46022 MR1014685 